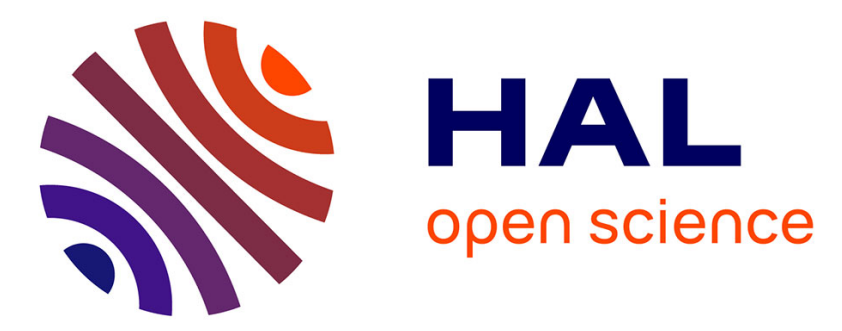

\title{
Anisotropic Swirling Surface Acoustic Waves from Inverse Filtering for On-Chip Generation of Acoustic Vortices
}

Antoine Riaud, Jean-Louis Thomas, Eric Charron, Adrien Bussonnière, Olivier Bou Matar, Michael Baudoin

\section{To cite this version:}

Antoine Riaud, Jean-Louis Thomas, Eric Charron, Adrien Bussonnière, Olivier Bou Matar, et al.. Anisotropic Swirling Surface Acoustic Waves from Inverse Filtering for On-Chip Generation of Acoustic Vortices. Physical Review Applied, 2015, 4 (3), pp.034004. 10.1103/PhysRevApplied.4.034004 . hal01398054

\section{HAL Id: hal-01398054 https://hal.science/hal-01398054}

Submitted on 24 Nov 2016

HAL is a multi-disciplinary open access archive for the deposit and dissemination of scientific research documents, whether they are published or not. The documents may come from teaching and research institutions in France or abroad, or from public or private research centers.
L'archive ouverte pluridisciplinaire HAL, est destinée au dépôt et à la diffusion de documents scientifiques de niveau recherche, publiés ou non, émanant des établissements d'enseignement et de recherche français ou étrangers, des laboratoires publics ou privés. 


\title{
Anisotropic Swirling Surface Acoustic Waves from Inverse Filtering for On-Chip Generation of Acoustic Vortices
}

\author{
Antoine Riaud, ${ }^{1,2}$ Jean-Louis Thomas, ${ }^{2}$ Eric Charron, ${ }^{2}$ Adrien Bussonnière, ${ }^{1}$ \\ Olivier Bou Matar, ${ }^{1}$ and Michael Baudoin ${ }^{1, *}$ \\ ${ }^{1}$ Institut d'Electronique, de Microélectronique et Nanotechnologie (IEMN), LIA LICS, \\ Université Lille 1 and EC Lille, UMR CNRS 8520, 59652 Villeneuve d'Ascq, France \\ ${ }^{2}$ Sorbonne Universités, UPMC Univ Paris 06, CNRS, UMR 7588, \\ Institut des NanoSciences de Paris (INSP), F-75005 Paris, France
}

(Received 29 April 2015; revised manuscript received 7 July 2015; published 15 September 2015)

\begin{abstract}
From radio-electronics signal analysis to biological sample actuation, surface acoustic waves (SAWs) are involved in a multitude of modern devices. However, only the most simple standing or progressive waves such as plane and focused waves have been explored so far. In this paper, we expand the SAW toolbox with a wave family named "swirling surface acoustic waves" which are the 2D anisotropic analogue of bulk acoustic vortices. Similarly to their 3D counterpart, they appear as concentric structures of bright rings with a phase singularity in their center resulting in a central dark spot. After the rigorous mathematical definition of these waves, we synthesize them experimentally through the inverse filtering technique revisited for surface waves. For this purpose, we design a setup combining arrays of interdigitated transducers and a multichannel electronic that enables one to synthesize any prescribed wave field compatible with the anisotropy of the substrate in a region called the "acoustic scene." This work opens prospects for the design of integrated acoustic vortex generators for on-chip selective acoustic tweezing.
\end{abstract}

DOI: $10.1103 /$ PhysRevApplied.4.034004

\section{INTRODUCTION}

Surface acoustic waves (SAWs) have become the cornerstone of microelectromechanical systems. SAWs not only are useful in delay lines and convolution filters [1] but can also monitor temperature variations, strain [2], magnetic fields $[3,4]$, and even chemical or biological composition [5,6]. More recently, the growing field of microfluidics has expressed tremendous interest towards SAWs $[7,8]$, due to their versatility for droplet actuation [9-12], atomization [13,14], jetting [15,16] or mixing [17], but also bubbles, particles, and cell manipulation and sorting [18-21]. Nevertheless, it is remarkable that all these functions rely on the most simple standing or progressive waves such as plane or focused waves.

At the end of the twentieth century, Durnin, Miceli, and Eberly [22] unveiled an exotic family of waves that do not diffract and can self-reconstruct. These waves propagate spinning around a phase singularity where destructive interferences lead to the total cancellation of the beam amplitude (Fig. 1). This concept was subsequently extended beyond optics [22-24] to acoustics [25,26] and even electronic wave functions [27-29]. In all cases, it is shown that vortical waves convey some pseudoangular momentum that exerts a measurable torque on lossy media.

*Corresponding author. michael.baudoin@univ-lille1.fr; http://films-lab.univ-lille1.fr
The dark core of these waves also plays a key role in trapping objects for optical or acoustic tweezers [30-32]. In the present study, we expand the SAW toolbox with a two-dimensional version of acoustic vortices, called for convenience swirling surface acoustic waves.

In two dimensions, swirling SAWs would appear as a dark spot circled by concentric bright rings of intense vibrations. It is tantamount to cloaking the focus of surface acoustic waves, allowing vigorous actuation of the direct neighborhood of fragile sensors. Furthermore, SAWs easily radiate from a piezoelectric solid to an adjacent liquid, simply by diving the transducer in the fluid. Swirling SAWs could therefore serve as integrated acoustic tweezers $[19,20]$. This would solve one of the major shortcomings of advanced pointwise acoustic tweezers [32-34], which are complex mechanical assemblies of numerous individual transducers, whereas the present swirling SAW generators are obtained by metal sputtering and photolithography on a single piezoelectric substrate. The radiation of swirling SAWs in adjacent liquid might also be used to monitor cyclonelike flows [35] in cavities by using a nonlinear effect called acoustic streaming. For these reasons, the present paper constitutes a first step towards more advanced acoustofluidic functionalities; the second steptransmission and propagation of swirling SAWs in the liquid phase-also holds several challenges and opportunities. For instance, it was previously observed in 3D optics that isotropic Bessel beams propagating in anisotropic 

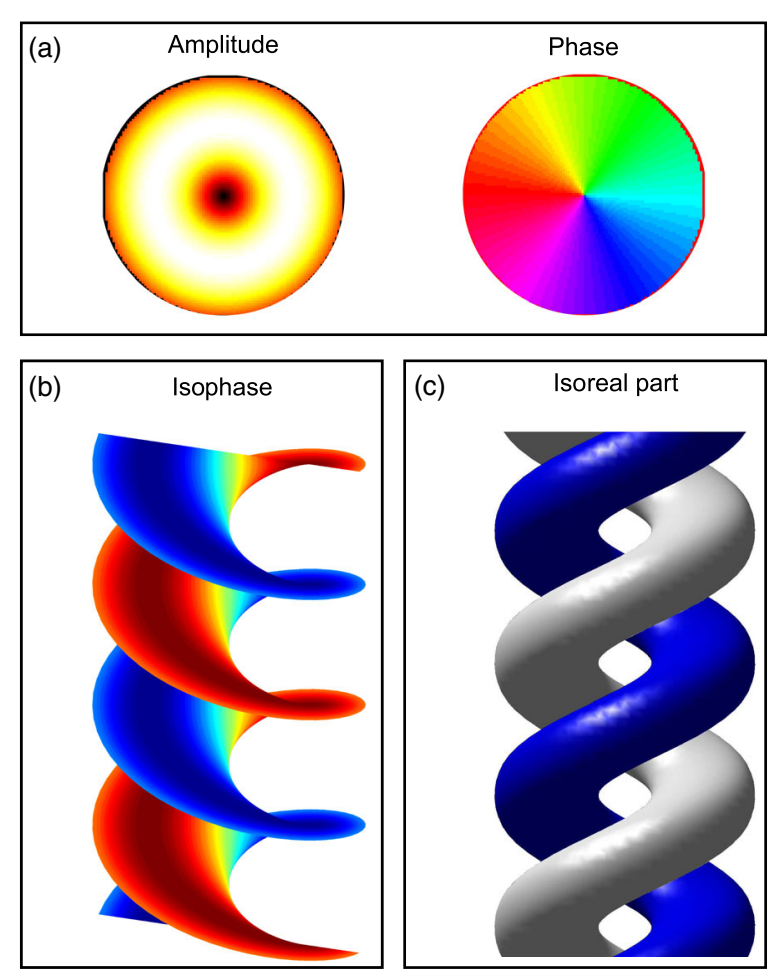

FIG. 1. A particular example of isotropic dark beams: the Bessel beams [Eq. (1) in Sec. II] with $l=1, k_{z}=1$, and $k_{r}=1$. (a) Beam cross section with a complex phase and amplitude. (b) Isophase surfaces at $l \theta-k_{z} z=0$ and $l \theta-k_{z} z=\pi$ in red and blue, respectively. (c) Isosurface of $\operatorname{Re}\left(W_{l}\right)=-0.3$ and +0.3 in blue and white, respectively.

media progressively lose coherence and disintegrate [36-39]. The converse phenomenon (disintegration of an anisotropic Bessel beam propagating in isotropic media) would offer a practical way to confine the acoustic vortex action to a bounded region of space. This subject will be covered in a dedicated report [40], while this one focuses on the definition and synthesis of swirling SAWs.

Since acoustic vortices have been known for a long time, the transition from $3 \mathrm{D}$ to $2 \mathrm{D}$ waves may appear as an insignificant step and one may wonder why it was not undertaken earlier. It is certainly the case if the wave propagates on a $2 \mathrm{D}$ isotropic medium such as perfectly sputtered piezoelectric thin films (AlN or $\mathrm{ZnO}$ ). However, the best piezoelectric coupling coefficients are obtained when using 2D anisotropic bulk piezoelectric crystals such as $\mathrm{LiNbO}_{3}$, which also happens to be the simplest and cheapest method to generate surface acoustic waves. Twenty years of intense research effort on anisotropic SAW focusing attest to the importance of these practical considerations [41-44]. Hence, in the following, we treat the more general case of an anisotropic medium. This involves two difficulties: First, although SAW synthesis is well mastered for single transducers radiating in specific directions of piezoelectric materials, the design of interdigitated transducer arrays (IDTAs) surrounding a control area is still challenging. Indeed, anisotropy considerably complicates the SAW propagation, leading to a direction-dependent wave velocity, coupling coefficient, and beam-stirring angle (noncollinear wave and energy vectors). Thanks to recent mathematical developments [45-48], SAW far-field propagation is better handled nowadays. Nevertheless, these methods require an accurate depiction of the target field in order to design the generator. The second difficulty is then to define exactly what a swirling SAW is, especially in an anisotropic medium. Since these waves are the fragile result of destructive interference, extreme care must be taken in computing their propagation.

In the present study, we use an adaptive field synthesis method in order to tackle the first issue. For this purpose, a sample of piezoelectric material is covered with a circular array of 32 independent transducers actuated by a programmable electronic. Then, its vibrations are monitored by a Michelson interferometer. The exact input is computed by an advanced calibration procedure called inverse filtering $[26,32,49]$.

Getting rid of the issue of emitter design, we efficiently focus on the definition of swirling surface acoustic waves. Our theoretical work is essentially guided by Laude, JerezHanckes, and Ballandras [48], who unveil and synthesize a zero-order anisotropic Bessel function. In a different context (multipole expansion of electromagnetic waves for numerical computation), Piller and Martin propose a comprehensive extension of Bessel functions to anisotropic media [50]. Our theoretical investigation, described in the first part of the paper, uses the concepts of slowness surface and angular spectrum to fill the gap between Piller and Martin's mathematical expression and surface acoustic waves. The next part of the paper describes our experimental setup, from the transducer design to the SAW measurements. The third part explains how we compute the IDTA signals in order to synthesize swirling SAWs. It provides the key steps of the inverse filtering method adapted to the propagation of surface acoustic waves. Finally, a fourth section exhibits some experimental swirling surface acoustic waves.

\section{DEFINITION OF AN ANISOTROPIC BESSEL FUNCTION}

A classical solution to the wave equation in isotropic medium is known as the Bessel beam:

$$
W_{l} e^{-i \omega t}=J_{l}\left(k_{r} r\right) e^{i l \theta+i k_{z} z-i \omega t}=W_{l}^{0} e^{i k_{z} z-i \omega t} .
$$

In this equation, $r, \theta, z, t, l, W_{l}, J_{l}, k_{r}, k_{z}, \omega$, and $W_{l}^{0}$ stand, respectively, for the axisymmetric coordinates, the time, the topological order, the complex wave-field value, the $l$ th-order Bessel function, the radial and axial parts of wave vector, the angular frequency, and the isotropic swirling surface acoustic wave complex value. 
The slowness surface and angular spectrum [51] constitute the basic blocks for building anisotropic wave fields. The main idea of these tools is to reduce the problem to a superposition of plane waves. For each single direction and frequency, we solve the 1D propagation equation, which reduces the partial differential equation to a set of ordinary differential equations, whose integration is straightforward. Hence, we first briefly review these concepts and then use them to derive a general 3D anisotropic Bessel beam. We eventually introduce the anisotropic swirling wave as a special case of an anisotropic vortex.

In the following, we work at a given frequency and omit the term $e^{-i \omega t}$ for clarity. In isotropic materials such as water, the wave speed of sound or light is independent of the direction of propagation. Consequently, the magnitude of the wave vector $k=2 \pi / \lambda$ is also a constant, and its locus versus the direction of propagation is a sphere called the slowness surface. Conversely, in the case of an anisotropic material, the wave speed depends on the direction, and so does the wave vector. In the reciprocal space of a 3D medium, we call $\Phi$ the azimuth and $\kappa_{z}$ the altitude in cylindrical coordinates, so the wave vector reads $\boldsymbol{k}\left(\phi, \kappa_{z}\right)$. The locus of this wave vector, still called the slowness surface, then results in nonspherical shapes depending on the anisotropy of the material [52]. Bessel beams propagate along a specific axis $z$. Consequently, discussions on the surface slowness often refer to $k_{r}\left(\phi, \kappa_{z}\right)$, the projection of $\mathbf{k}$ on the plane normal to the propagation axis. The axial and radial components of the wave vector $k_{z}$ and $k_{r}$, respectively, are linked by the directionwise dispersion relation:

$$
k_{z}\left(\phi, \kappa_{z}\right)^{2}+k_{r}\left(\phi, \kappa_{z}\right)^{2}=\frac{\omega^{2}}{c\left(\phi, \kappa_{z}\right)^{2}} .
$$

In Eq. (2), we write the coordinates in the reciprocal space $\kappa_{i}$ to distinguish them from $k_{i}$, which refer to the dispersion relation of the wave and are given physical quantities. For instance, $\kappa_{z}$ can take any value, whereas $k_{z}$ is defined only in a closed interval $\left(k_{z} \in[-\omega / c,+\omega / c]\right.$ for an isotropic medium).

The angular spectrum is a multidimensional generalization of the Fourier transform. Since Fourier's pioneering work, it is known that any field can be resolved into a sum of sinusoidal functions. The angular spectrum is a recursive application of the Fourier transform over all the dimensions of the medium:

$$
\begin{aligned}
f(x, y, z)= & \int_{-\infty}^{+\infty} \int_{-\infty}^{+\infty} \int_{-\infty}^{+\infty} F\left(\kappa_{x}, \kappa_{y}, \kappa_{z}\right) e^{i \kappa_{x} x} d \kappa_{x} e^{i \kappa_{y} y} \\
& \times d \kappa_{y} e^{i \kappa_{z} z} d \kappa_{z} .
\end{aligned}
$$

We can rearrange the terms in the exponential in order to get $\exp \left[i\left(\kappa_{x} x+\kappa_{y} y+\kappa_{z} z\right)\right]$ such that Eq. (3) can be interpreted as a sum of plane waves. This means that any physical field in the medium at a given frequency can be seen as a combination of plane waves and therefore must satisfy the dispersion relation or, equivalently, lie on the slowness surface. In this regard, the slowness surface provides a frame for the wave landscape, and choosing the angular spectrum $F\left(\kappa_{x}, \kappa_{y}, \kappa_{z}\right)$ amounts to applying the color (complex phase and amplitude) on this frame.

If we express the previous angular spectrum not in Cartesian coordinates but in cylindrical ones, we get

$$
\begin{aligned}
f(r, \theta, z)= & \int_{-\infty}^{+\infty} \int_{-\pi}^{+\pi} \int_{0}^{+\infty} F\left(\kappa_{r}, \phi, \kappa_{z}\right) e^{i \kappa_{r} r \cos (\phi-\theta)} \kappa_{r} d \kappa_{r} \\
& \times d \phi e^{i \kappa_{z} z} d \kappa_{z} .
\end{aligned}
$$

In this expression, the variables $\kappa_{r}, \phi$, and $\kappa_{z}$ refer to the spectral domain, whereas $r, \theta$, and $z$ belong to the spatial one. In order to satisfy the dispersion relation, we know that $F$ must vanish anywhere except on the slowness surface, so $F\left(\kappa_{r}, \phi, \kappa_{z}\right)=h\left(\phi, \kappa_{z}\right) \delta\left[\kappa_{r}-k_{r}\left(\phi, \kappa_{z}\right)\right]$, with $k_{r}$ the magnitude of the wave vector in the $(x, y)$ plane and $h$ an arbitrary function of $\phi$ and $\kappa_{z}$. This reduces the set of waves that can be created in the medium:

$$
\begin{aligned}
f(r, \theta, z)= & \int_{-\infty}^{+\infty} \int_{-\pi}^{+\pi} h\left(\phi, \kappa_{z}\right) e^{i k_{r}\left(\phi, \kappa_{z}\right) r \cos (\phi-\theta)} \\
& \times k_{r}\left(\phi, \kappa_{z}\right) d \phi e^{i \kappa_{z} z} d \kappa_{z} .
\end{aligned}
$$

At a given $\kappa_{z}$, the integral in Eq. (5) is the product of two terms: The first one $e^{i k_{r}\left(\phi, \kappa_{z}\right) r \cos (\phi-\theta)}$ can be reduced to a sum of plane waves thanks to Jacobi-Anger expansion, while the second one $h k_{r}$ provides the color of each of these plane waves.

We construct anisotropic Bessel functions by splitting the wave angular spectrum in a $\kappa_{z}$-independent part and extracting its coefficients. Since $\phi$ is the azimuth, it is a periodic function and we can expand $h k_{r}$ in Fourier series: $h k_{r}=\sum_{-\infty}^{+\infty} a_{l}\left(\kappa_{z}\right) e^{i l \phi}$. We then get

$$
\begin{aligned}
f(r, \theta, z)= & \int_{-\infty}^{+\infty} \sum_{l=-\infty}^{+\infty} a_{l}\left(\kappa_{z}\right) e^{i \kappa_{z} z} \\
& \times \int_{-\pi}^{+\pi} e^{i l \phi+i k_{r}\left(\phi, \kappa_{z}\right) r \cos (\phi-\theta)} d \phi d \kappa_{z} .
\end{aligned}
$$

As mentioned earlier, the integral can be interpreted as a sum over all the $\kappa_{z}$ of some elementary functions. In these functions, $\kappa_{z}$ appears as a parameter instead of a variable.

In order to highlight what in this expansion may be reminiscent of a Bessel, we need to write the integral expression of the Bessel function:

$$
J_{l}(x)=\frac{1}{2 \pi} \int_{-\pi}^{+\pi} e^{i l \eta-i x \sin (\eta)} d \eta .
$$

A trivial change of variable $\eta=\phi-\theta-\pi / 2$ yields 


$$
J_{l}(x)=\frac{1}{2 \pi i^{l}} \int_{-\pi}^{+\pi} e^{i l(\phi-\theta)+i x \cos (\phi-\theta)} d \phi .
$$

We combine Eqs. (1) and (8) to get the isotropic swirling SAW:

$$
W_{l}^{0}(r, \theta)=\frac{1}{2 \pi i^{l}} \int_{-\pi}^{+\pi} e^{i l \phi+i k_{r} r \cos (\phi-\theta)} d \phi
$$

By analogy with the isotropic equation, we define an anisotropic swirling wave with a given $\kappa_{z}=k_{z}$ as

$$
\mathcal{W}_{l}^{0}(r, \theta)=\frac{1}{2 \pi i^{l}} \int_{-\pi}^{+\pi} e^{i l \phi+i k_{r}\left(\phi, k_{z}\right) r \cos (\phi-\theta)} d \phi .
$$

SAWs appear as a specialization of Eq. (10) to waves that propagate only along the substrate surface, leading to $k_{z}=0$. Interestingly, the beam in Eq. (10) shares a common mathematical expression with the electromagnetic multipole used by Piller and Martin [50] for solving anisotropic scattering problems, which augurs that such an anisotropic Bessel beam might be extremely widespread in nature.

Incidentally, any wave in an anisotropic medium can be written as a combination of anisotropic Bessel beams $\mathcal{W}_{l}=\mathcal{W}_{l}^{0} e^{i \kappa_{z} z}$ :

$$
f(r, \theta, z)=\int_{-\infty}^{+\infty} \sum_{l=-\infty}^{+\infty} a_{l}\left(\kappa_{z}\right) 2 \pi i^{l} \mathcal{W}_{l}^{0}\left(r, \theta, \kappa_{z}\right) e^{i \kappa_{z} z} d \kappa_{z}
$$

In the rest of the paper, we use inverse filtering to generate anisotropic swirling SAW $\mathcal{W}_{l}^{0}$ on the surface of an anisotropic piezoelectric crystal.

\section{EXPERIMENTAL SETUP}

The experimental setup is designed to be as versatile as possible, in order to allow generating a wide variety of waves on an area called the acoustic scene. Starting from an $\mathrm{X}$-cut lithium niobate crystal, 32 unidirectional interdigitated transducers (SPUDT IDTs) are deposited on its periphery (see Fig. 2). In order to widen the range of possible acoustic fields, every spot on the scene should be illuminated by all the transducers. This spatial coverage should be as uniform as possible on the acoustic scene. It is achieved by using IDTs with narrow apertures and disposing them remotely from the acoustic scene to promote diffraction. Furthermore, since any wave can be described as a combination of plane waves, it is essential to generate waves from a wide span of directions. Hence, the quality of the wave-field synthesis critically depends on the span of plane waves provided by the source array in terms of the incident angle, which is the angular spectrum coverage. The best way to achieve such optimal coverage is therefore

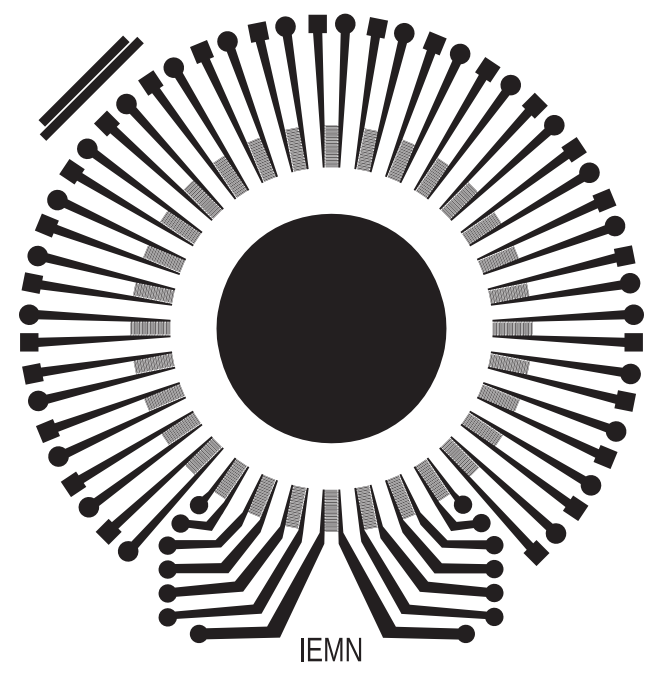

FIG. 2. Interdigitated transducer array used for generating the surface acoustic waves. The central black disk (25-mm diameter) is a gold layer acting as a mirror for interferometric measurements and materializes the maximum extent of the acoustic scene. Vector format image (available online) is used to visualize the fine structure of the electrodes.

to gather many sources from all directions and dispose them radially around a target spot which will be the acoustic scene. These notions of optimal coverage are detailed further in the next section and in the Appendix.

In order to measure the wave field on the acoustic scene, we place the sample under the motorized arm of a polarized Michelson interferometer (Fig. 3). The poor reflection coefficient of lithium niobate is significantly increased by the deposition of a thin layer of gold on the acoustic scene (approximately $200 \mathrm{~nm}$ ).

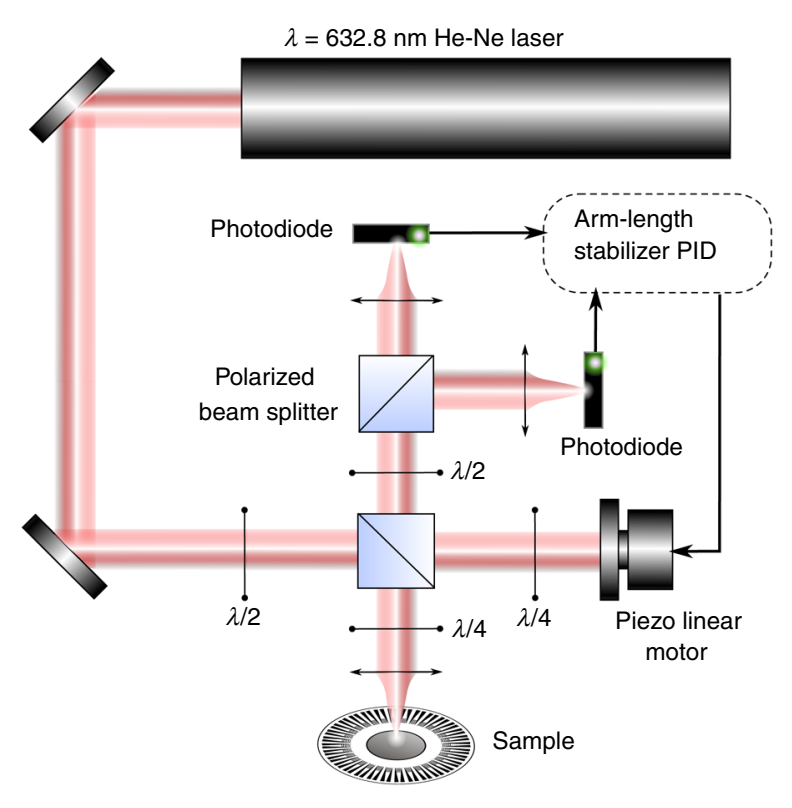

FIG. 3. Polarized Michelson interferometer used for scanning the displacement field associated with surface acoustic waves. 
(a)

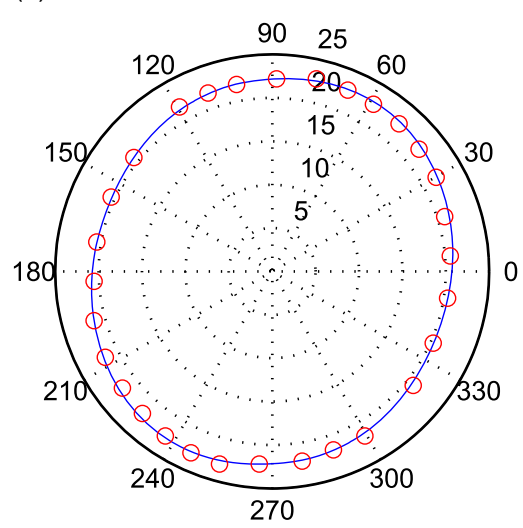

(b)

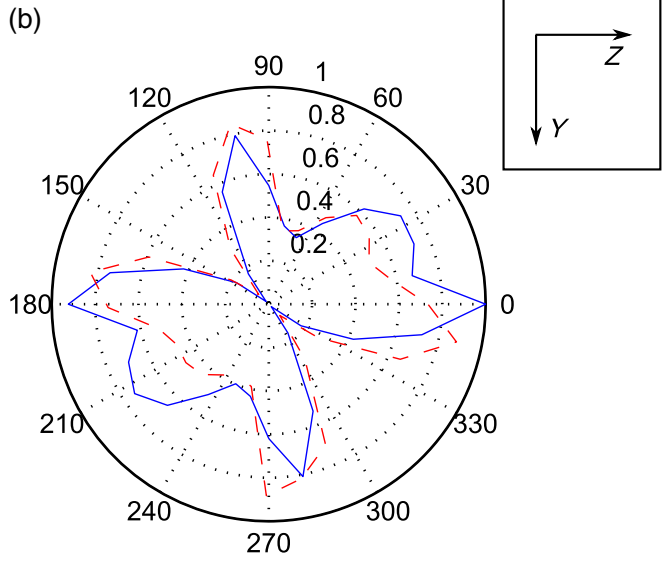

FIG. 4. (a) Theoretical slowness contour ( $\mathrm{rad} / \mathrm{mm}$, blue solid line) under a very thin gold layer [53] versus the experimentally measured one (red circles). (b) Normalized SAW vertical displacement magnitude at the center of the substrate (theoretical, blue solid line; experimental, red dashed line). The max displacement magnitude is $1.8 \mathrm{~nm}$. Inset: Crystallographic axes.
During the design of the IDTs, special care is given to the anisotropy of the lithium niobate substrate. Indeed, IDTs are high-quality spatiotemporal resonant elements with a spatial period equal to the wavelength. Any deviation from the narrow resonant bandwidth results in a very significant loss of efficiency [1]. We plot in Fig. 4(a) the slowness contour of lithium niobate measured on the gold layer at the working frequency of $12 \mathrm{MHz}$ and compare it to theoretical predictions [53]. The two directions with the lowest SAW magnitude are missing in the experimental data set. The vertical wave motion at the center of substrate is recorded experimentally for each transducer and plotted in Fig. 4(b). The butterfly pattern unambiguously reflects the substrate anisotropy. It is the combination of piezoelectric coupling and beam-stirring effects and can be computed using the Green functions introduced thereafter.

The knowledge of the dispersion relation provides the wave field radiated by a single point source $[42,48]$ :

$G(r, \theta) \simeq A a(\bar{\phi}) \frac{\exp \left\{-i \omega r h(\bar{\phi})-i \frac{\pi}{4} \operatorname{sgn}\left[h^{\prime \prime}(\bar{\phi})\right]\right\}}{\sqrt{\omega r\left|h^{\prime \prime}(\bar{\phi})\right|}}$

with $a(\phi)$ the coupling coefficient between the field to measure and the electrical potential (obtained when solving the SAW equations $[53,54]), \bar{\phi}(\phi)$ the beam-stirring angle, $h(\phi)=\cos (\phi-\theta) k(\phi) / \omega(\phi)$, and $h^{\prime \prime}=d^{2} h[\bar{\phi}(\phi)] / d \phi^{2}$ related to the focusing factor. The beam-stirring angle is the solution of $h^{\prime}(\bar{\phi})=0$.

Thanks to the superposition principle, we can use the Green function in Eq. (12) to compute the acoustic field radiated by our emitter arrays. The predictions are compared to experiments in Fig. 5. Anisotropy strongly affects the SAW propagation, as we can observe beam widening [(a),(b)], focusing [(c),(d)], and stirring [(e),(f)] depending on the beam direction. Despite a general good agreement between numerical and experimental results, this 2D Green function approach also exhibits some intrinsic limitations.
For instance, the lobes on the SAW beam in Fig. 5(b)confirmed by Fig. 4(b) - are not predicted theoretically. Given the important assumptions of 2D half-space, we believe the suspicious SAW is actually a leaky SAW, and it
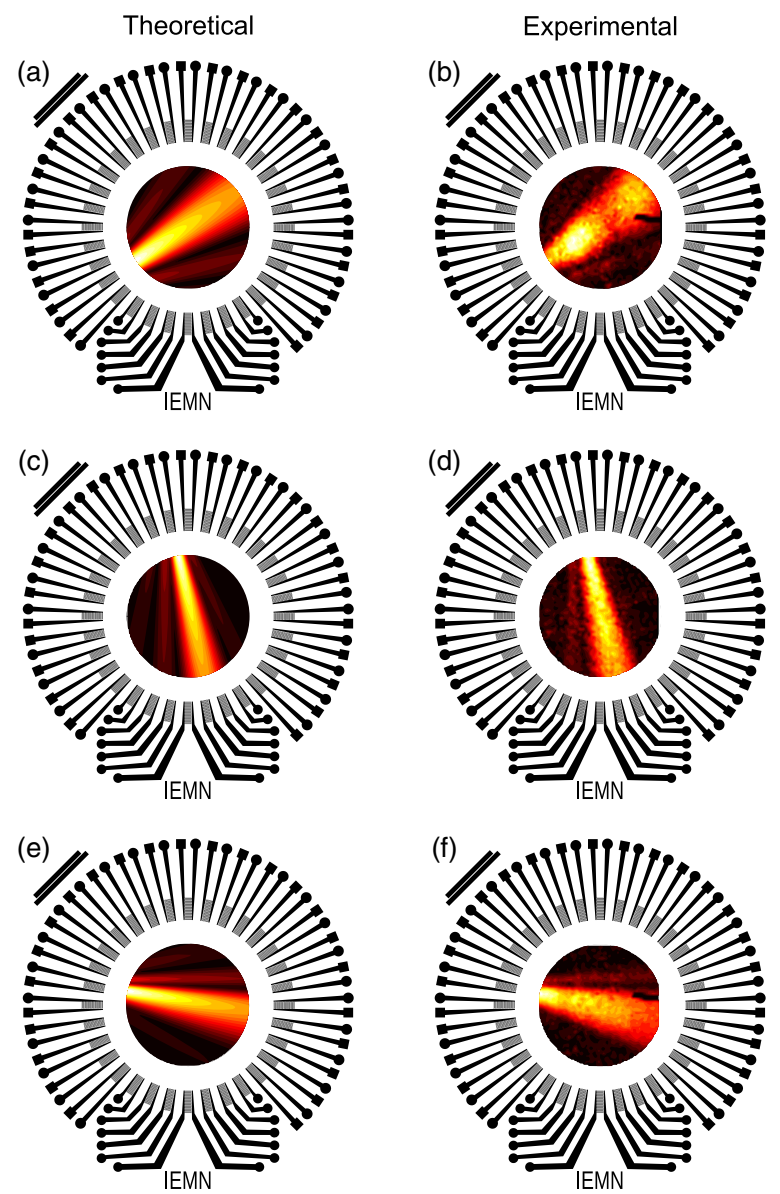

FIG. 5. Influence of anisotropy on the propagation of SAWs generated by single electrodes. (a), (c), (e) Theoretical predictions; (b), (d), (f) experimental measurements. (a), (b) Beam widening; (c), (d) beam focusing; (e), (f) beam stirring. Color represents the beam relative intensity over the substrate and is not indicative of the ratio of intensity between two different transducers. 
generates a bulk acoustic wave which bounces between the two faces of the substrate. All these issues of the anisotropic piezoelectric coupling coefficient, beam stirring, and power lobes are significantly alleviated by inverse filtering.

A wide-band high-power multichannel fieldprogrammable gate array (FPGA) (Lecoeur Electronics) powers the 32 emitters with tailored numerical input. The input is specific to each desired wave field and designed through the inverse filtering method.

\section{INVERSE FILTERING THEORY}

Inverse filtering [49] is a very general technique for analyzing or synthesizing complex signals that propagate through arbitrary linear medium. This method is especially suited for prototyping, because, given a set of independent programmable sources, it finds the optimal input signal to get a target wave field. When used for this purpose, it is similar to computer-generated holography in optics [55].

The method proceeds in four distinct stages (see Fig. 6): (i) calibration of the transducers, (ii),(iii) computation of the optimal input, and (iv) actuation of the sound sources according to the optimal input.

In the current system, we use a set of 32 emitters and an arbitrary number of control points evenly distributed on the acoustic scene. Their density is governed by the Shanon principle: The distance between two points should not exceed $\lambda / 2$. In our acquisition, we use a step of $\lambda / 10=30 \mu \mathrm{m}$. Moving the arm of the interferometer, we are able to reach individually each of these measurement points. If we call $e_{i}$ the temporal input of emitter $i$ and $s_{j}$ the temporal output of control point $j$ located on $\left\{x_{j}, y_{j}\right\}$, we have for any linear medium

$$
s_{j}=\sum_{i} h_{i j} * e_{i},
$$

where $*$ refers to the convolution product and $h_{i j}$ is the time response at control point $j$ to an impulse input at emitter $e_{i}$. In the spectral domain, $H_{i j}=\mathcal{F}\left(h_{i j}\right)$ is the Fourier transform of the transfer function at control point $j$ of emitter $i$ and includes the propagation of the wave in the medium. Using the matrix formalism, things get even simpler:

$$
|\boldsymbol{S}\rangle=\boldsymbol{H}|\boldsymbol{E}\rangle .
$$

In case the transfer matrix is square and well conditioned, it can be inverted to determine the optimal input $|\boldsymbol{E}\rangle$ from a desired output $|\boldsymbol{S}\rangle$. However, the number of independent sources and control points is not necessarily the same, so $\boldsymbol{H}$ is generally not square and often ill conditioned. In the Appendix, we explain the reasons for this ill conditioning from the perspective of the angular spectrum and provide guidelines to minimize it. Although inverse filtering was previously shown to be among the most accurate ones for generating acoustic vortices in 3D isotropic media [26], it was never used for 2D anisotropic media. In previous configurations, the target field is a surface and has a smaller dimensionality (2D) than the propagative medium (3D), whereas the current setup enforces a target field of the same dimensionality as the propagative medium (both 2D). Hence, in the current experiment, the knowledge of the target field explicitly sets an angular spectrum for the propagation medium. However, the wave field in the same medium must fulfill the dispersion relation. Hence, the impulse response matrix is zero everywhere outside the slowness surface. In practice, however, small amplitude noise will always fill these nonpropagative regions. If the target field contains any point outside the slowness surface, the inversion operator would be mistaken as it would rely on the measurement noise to achieve an optimal signal synthesis. Hence, it is essential to define the target field along the slowness curve and resample the impulse

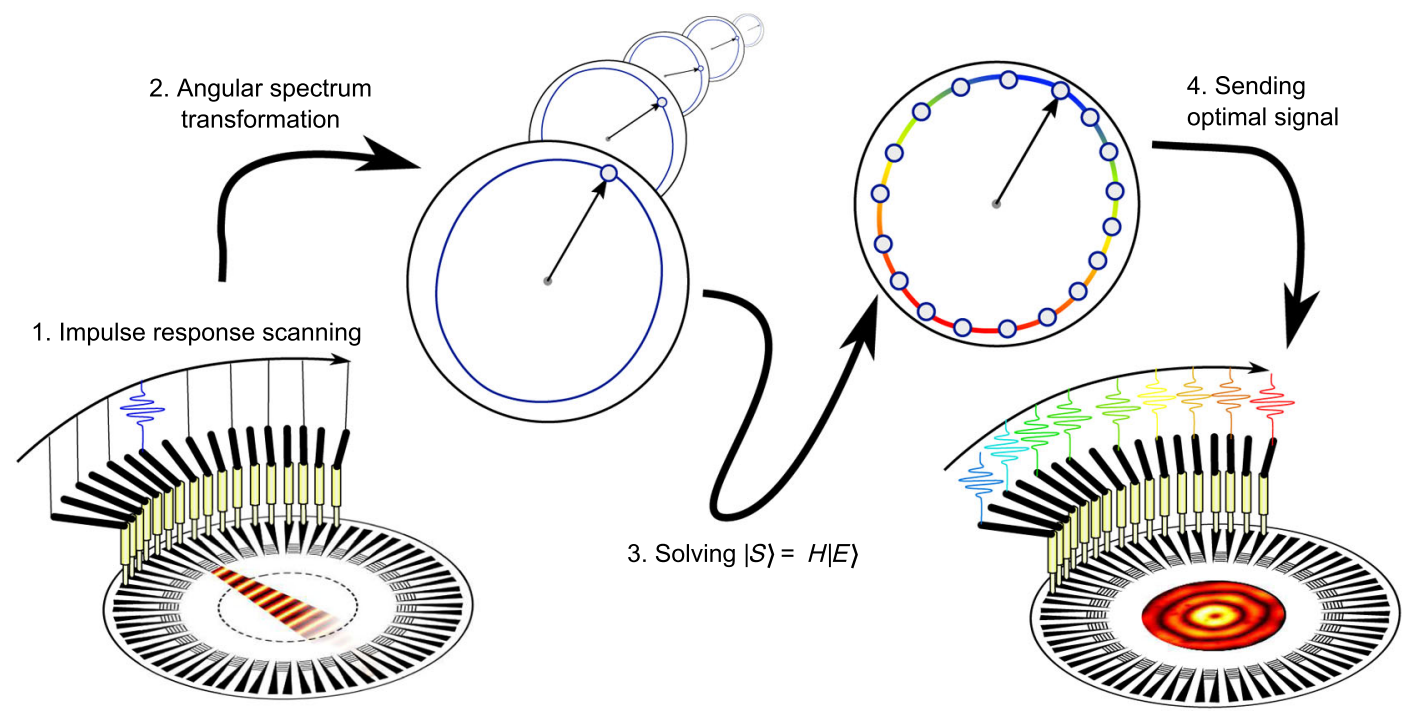

FIG. 6. Inverse filtering flowchart. Inverse filtering happens in four steps. (i) Recording of the spatial impulse response ( $H$ matrix) for all transducers. (ii) Transformation of the $H$ matrix from a spatial to spectral domain, where the response is sharper. (iii) Computation of the optimal input $|\boldsymbol{E}\rangle$ for a desired output $|S\rangle$ by pseudoinversion of the matrix $\boldsymbol{H}$. (iv) Generation of the signal from optimal input $|\boldsymbol{E}\rangle$. 
response matrix in the same subset of the reciprocal space. We call this method spectral inverse filtering.

As soon as the value of $|\boldsymbol{E}\rangle$ has been computed, the timedependent input is obtained by inverse Fourier transform and sent to the FPGA amplifier to generate the wave field.

\section{EXPERIMENTAL RESULTS}

Bessel beams draw large interest for three main reasons: They do not diffract [22], they carry a pseudo-orbital momentum [23,56,57], and they exhibit a dark core (for nonzero order) $[32,33]$. In addition to these reasons, the zero-order Bessel beam is the optimal beam focusing for a given aperture [42]. In the following section, we start by synthesizing a focused surface acoustic wave $\mathcal{W}_{0}^{0}$ and then some simple first-order swirling SAW $\mathcal{W}_{1}^{0}$. We seize this opportunity to show the phase singularity and the associated dark spot. The size of the dark spot can easily be tuned, simply by changing the topological charge $l$, which is done in the third example with seventh-order swirling SAWs.

The zero-order focused $\mathcal{W}_{0}^{0}$ Bessel wave phase and amplitude are traced in Fig. 7. It appears that theoretical fields and experimental ones are quite similar. In practice, we have to limit the voltage amplitude of our instrument to about $10 \%$ in order to get a linear response of the interferometer (the upper bond is about $40 \mathrm{~nm}$ ). For high actuation power, we estimate the displacement amplitude based on the second bright ring. When setting the voltage to about $50 \%$, we achieve a displacement amplitude of nearly $180 \mathrm{~nm}$.
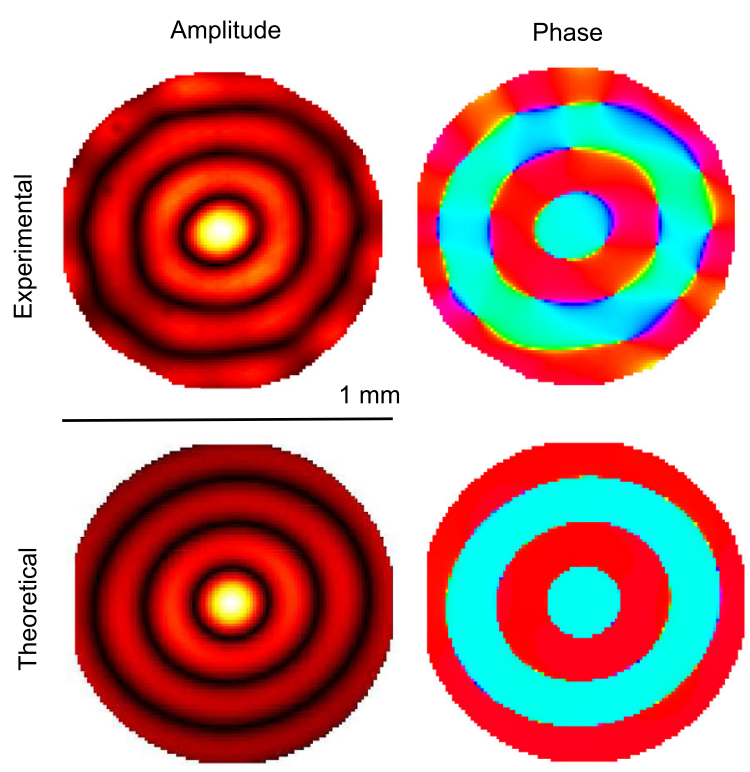

FIG. 7. Experimental and theoretically predicted zero-order focused $\mathcal{W}_{0}^{0}$ Bessel wave phase and amplitude. The maximum experimental displacement is $40 \mathrm{~nm}$.
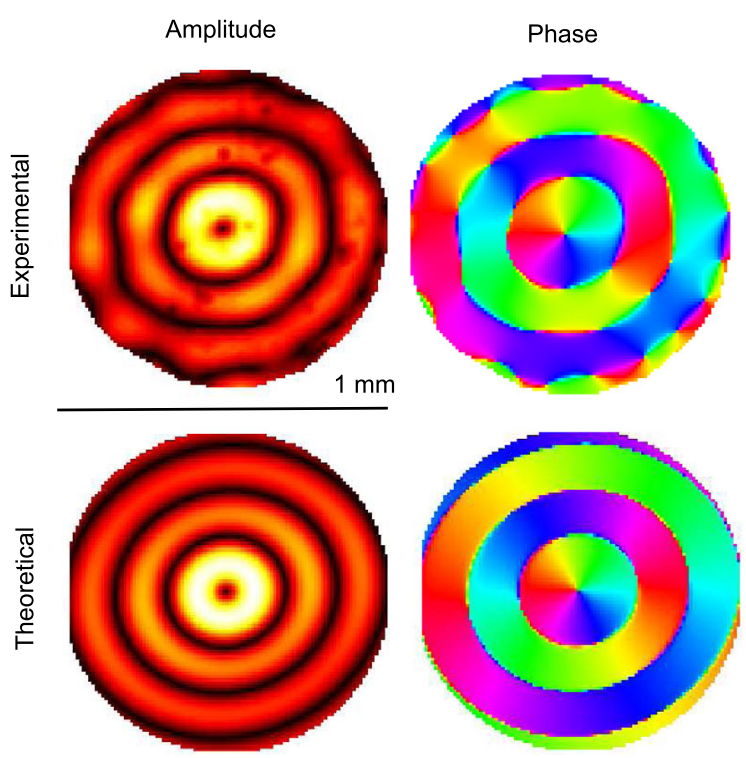

FIG. 8. Experimental and theoretically predicted first-order $\mathcal{W}_{1}^{0}$ Bessel wave phase and amplitude. The maximum experimental displacement is $36 \mathrm{~nm}$.

Figure 8 represents the first-order dark beam $\mathcal{W}_{1}^{0}$ phase and amplitude. A dark core of zero amplitude with a diameter of $50 \mu \mathrm{m}$ is clearly visible at the center of the vortex and matches with a phase singularity. This area is contrasted by very bright concentric rings. Despite some blur in the experimental measurements, a good matching between theoretical and experimental vortices is achieved on both the shape and phase.

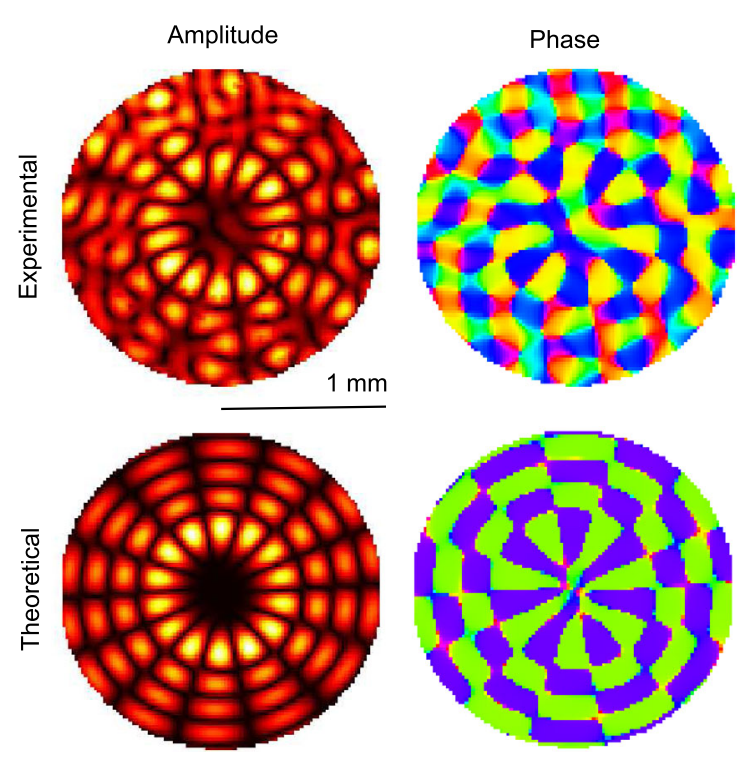

FIG. 9. Experimental and theoretical predictions of the combination of two seventh-order vortices $\mathcal{W}_{ \pm 7}^{0}$ of opposite charge. The maximum experimental displacement is $25 \mathrm{~nm}$. 
Swirling SAWs might be useful as integrated transducers for acoustic tweezers or micropumps. Tuning the topological order is essential to these applications for two reasons: It enlarges the first bright ring of the vortex [Olver formula [58] in Eq. (15)], and it increases the pseudoangular momentum of the wave [56]. The second effect itself generates acoustic streaming with an azimuthal flow velocity proportional to the topological charge [35,57]:

$$
j_{l, 1}^{\prime}=l\left(1+0.809 \times l^{-2 / 3}\right)+O\left(l^{-7 / 3}\right) .
$$

In this last example, we suggest a way to increase the ring radius while maintaining zero azimuthal streaming and keep working at the resonance frequency of the electrodes. When two isotropic vortices of opposite charge are combined, they result in a circular stationary wave pattern. In the present case, we sum two seventh-order contrarotating acoustic vortices $\mathcal{W}_{7}^{0}+\mathcal{W}_{-7}^{0}$. The resulting field, shown in Fig. 9, exhibits a dark core with a diameter of about $500 \mu \mathrm{m}$ circled by a crown made of 14 extrema of amplitude.

\section{CONCLUSION}

In this report, we propose an anisotropic SAW version of acoustic vortices, labeled swirling surface acoustic waves. This implies solving two difficulties: First, the generator has to be designed to accommodate anisotropic propagation, and second, we need to define accurately what are swirling SAWs. The first problem is alleviated using a programmable array of transducers controlled by a twodimensional spectral inverse filter, while the solution of the second problem confirms earlier theoretical predictions. We synthesize swirling SAWs of different topological charges and large magnitude of displacement. This successful generation provides a pathway for integrated acoustic vortex generators on anisotropic substrates. Furthermore, since these beams are expected to radiate in any adjacent fluid, photolithography fabricated swirling SAW transducers constitute a step towards a credible alternative to the current complicated acoustic tweezer devices made of mechanical assemblies of individual transducers. Beyond the specificities of acoustics, Bessel functions are very widespread in nature, and anisotropic Bessels may offer analytical solutions for a broad class of linear anisotropic problems.

\section{ACKNOWLEDGMENTS}

The authors thank Silbe Majrab, who developed and constructed the piezoregulator of the interferometer, and Rémi Marchal, who shared with us precious advice on the art of Michelson interferometry. This work is supported by ANR Project No. ANR-12-BS09-0021-01 and ANR-12BS09-0021-02, and Région Nord Pas de Calais.

\section{APPENDIX: OPTIMAL CONDITIONING OF INVERSE FILTERING}

Inverse filtering is a very versatile method to synthesize an optimal target field from a given number of transducers. As stated in Sec. IV, the method is not exempt from poor conditioning, which would result in large errors in the synthesized field, but some guidelines can significantly improve the quality of the field synthesis. The poor conditioning of inverse filtering has two roots: (i) spectral outliers and (ii) redundant sources.

\section{Spectral outliers}

At a given frequency, the wave field must fulfill the dispersion relation, which is to have its angular spectrum lying on its slowness surface. When the acoustic scene is a surface (in 2D) or a volume (in 3D), this condition exactly happens. However, experimentally, there is always some noise introduced in the impulse response matrix, making it full rank (any spatial frequency can be created provided there is enough input power). Consequently, a first regularization is to remove the spectral outliers by sampling the target field not on a spatial manifold but on a spectral one and along the slowness surface.

Nevertheless, if the acoustic scene is a line (in 2D) or a surface (in 3D) as in previous implementations [26,49], the spectral condition is relaxed. Indeed, the angular spectrum of the target field is only partially known due to the projection of the field along the line or the surface. In 3D, for instance, if the synthesis happens on an $\{x, y\}$ plane, the system knows the values of $k_{x}$ and $k_{y}$ but ignores the ones of $k_{z}$, which can then be freely chosen as long as the dispersion relation is fulfilled. In an isotropic medium, this results in $k_{z}= \pm \sqrt{\omega^{2} / c^{2}-k_{x}{ }^{2}-k_{y}{ }^{2}}$. Note that, in any case, $\omega^{2} / c^{2}>k_{x}{ }^{2}+k_{y}{ }^{2}$, which is the diffraction limit. Hence, spectral outliers in this synthesis appear beyond the $\lambda / 2$ boundary.

A third example of spectral outliers is provided by piezoelectric generation on monocrystals. These substrates often exhibit a direction where the piezoelectric coupling coefficient sharply drops to zero. When this happens, no acoustic waves can be generated from this orientation, and the associated angular spectrum coverage is barely zero. Once again, sampling the signal in the spectral space and excluding the zero-coupling directions avoids these outliers and allows an accurate synthesis.

\section{Redundant sources}

In practice, many transducers are used to ensure an efficient spectral coverage. Above this threshold, adding even more actuators may result in poorer synthesis quality [49]. Indeed, from the inverse filtering perspective, sound sources act like a family of vectors to combine in order to build a target field. When two sources are redundant, the 
inversion operator can take any linear combination of them, and this indetermination is solved by comparing the measurement noise associated with each source. A smart way is therefore to regularize the reduced impulse response matrix obtained after removing the spectral outliers. The regularization can be achieved by a singular value decomposition. If two transducers are redundant, they split in a singular value very close to zero and another one much more regular. By knowing the signal-to-noise ratio, it is then possible to discriminate which singular values originate from noise and which do not [49].

[1] Daniel Royer and Eugène Dieulesaint, Elastic Waves in Solids 2, Generation, Acousto-Optic Interactions, Applications (Springer, New York, 1996), Chap. 2.1.3.

[2] A. Pohl and F. Seifert, in Proceedings of the Instrumentation and Measurement Technology Conference (IEEE, New York, 1996), Vol. 2, pp. 1465-1468.

[3] Odilon D. D. Couto, R. Hey, and P. V. Santos, Spin dynamics in (110) GaAs quantum wells under surface acoustic waves, Phys. Rev. B 78, 153305 (2008).

[4] Jörg B. Kinzel, Daniel Rudolph, Max Bichler, Gerhard Abstreiter, Jonathan J. Finley, Gregor Koblmüller, Achim Wixforth, and Hubert J. Krenner, Directional and dynamic modulation of the optical emission of an individual GaAs nanowire using surface acoustic waves, Nano Lett. 11, 1512 (2011).

[5] A. Pohl, A review of wireless SAW sensors, IEEE Trans. Ultrason. Ferroelectr. Freq. Control 47, 317 (2000).

[6] David Stephen Ballantine, Acoustic Wave Sensors: Theory, Design, and Physico-Chemical Applications (Academic, New York, 1997).

[7] Trung Dung Luong and Nam Trung Nguyen, Surface acoustic wave driven microfluidics-A review, Micro Nanosyst. 2, 217 (2010).

[8] Leslie Y. Yeo and James R. Friend, Surface acoustic wave microfluidics, Annu. Rev. Fluid Mech. 46, 379 (2014).

[9] Thomas Franke, Adam R. Abate, David A. Weitz, and Achim Wixforth, Surface acoustic wave (SAW) directed droplet flow in microfluidics for PDMS devices, Lab Chip 9, 2625 (2009).

[10] A. Wixforth, C. Strobl, C. Gauer, A. Toegl, J. Scriba, and Z. Guttenberg, Acoustic manipulation of small droplets, Anal. Bioanal. Chem. 379, 982 (2004).

[11] A. Renaudin, P. Tabourier, V. Zhang, J. C. Camart, and C. Druon, SAW nanopump for handling droplets in view of biological applications, Sens. Actuators, B 113, 389 (2006).

[12] M. Baudoin, P. Brunet, O. Bou Matar, and E. Herth, Low power sessile droplets actuation via modulated surface acoustic waves, Appl. Phys. Lett. 100, 154102 (2012).

[13] K. Chono, N. Shimizu, Y. Matsui, J. Kondoh, and S. Shiokawa, Development of novel atomization system based on SAW streaming, Jpn. J. Appl. Phys. 43, 2987 (2004).

[14] A. Qi, L. Y. Yeo, and J. R. Friend, Interfacial destabilization and atomization driven by surface acoustic waves, Phys. Fluids 20, 074103 (2008).
[15] S. Shiokawa, Y. Matsui, and T. Ueda, Study of SAW streaming and its application to fluid device, Jpn. J. Appl. Phys. 29, 137 (1990).

[16] M. K. Tan, J. R. Friend, and L. Y. Yeo, Interfacial Jetting Phenomena Induced by Focused Surface Vibrations, Phys. Rev. Lett. 103, 024501 (2009).

[17] T. Frommelt, M. Kostur, M. Wenzel-Schäfer, P. Talkner, P. Hänggi, and A. Wixforth, Microfluidic Mixing via Acoustically Driven Chaotic Advection, Phys. Rev. Lett. 100, 034502 (2008).

[18] Mikkel Settnes and Henrik Bruus, Forces acting on a small particle in an acoustical field in a viscous fluid, Phys. Rev. E 85, 016327 (2012).

[19] Xiaoyun Ding, Sz-Chin Steven Lin, Brian Kiraly, Hongjun Yue, Sixing Li, I-Kao Chiang, Jinjie Shi, Stephen J. Benkovic, and Tony Jun Huang, On-chip manipulation of single microparticles, cells, and organisms using surface acoustic waves, Proc. Natl. Acad. Sci. U.S.A. 109, 11105 (2012).

[20] S. B. Q. Tran, P. Marmottant, and P. Thibault, Fast acoustic tweezers for the two-dimensional manipulation of individual particles in microfluidic channels, Appl. Phys. Lett. 101, 114103 (2012).

[21] Adrien Bussonnière, Yannick Miron, Michaël Baudoin, Olivier Bou Matar, Michel Grandbois, Paul Charette, and Alan Renaudin, Cell detachment and label-free cell sorting using modulated surface acoustic waves (SAWs) in droplet-based microfluidics, Lab Chip 14, 3556 (2014).

[22] J. Durnin, J. J. Miceli, and J. H. Eberly, Diffraction-Free Beams, Phys. Rev. Lett. 58, 1499 (1987).

[23] L. Allen, M. W. Beijersbergen, R. J. C. Spreeuw, and J. P. Woerdman, Orbital angular momentum of light and the transformation of Laguerre-Gaussian laser modes, Phys. Rev. A 45, 8185 (1992).

[24] H. He, M. E. J. Friese, N. R. Heckenberg, and H. Rubinsztein-Dunlop, Direct Observation of Transfer of Angular Momentum to Absorptive Particles from a Laser Beam with a Phase Singularity, Phys. Rev. Lett. 75, 826 (1995).

[25] B. T. Hefner and P. L. Marston, An acoustical helicoidal wave transducer with applications for the alignment of ultrasonic and underwater systems, J. Acoust. Soc. Am. 106, 3313 (1999).

[26] R. Marchiano and J.-L. Thomas, Synthesis and analysis of linear and nonlinear acoustical vortices, Phys. Rev. E 71, 066616 (2005).

[27] Vincenzo Grillo, Gian Carlo Gazzadi, Erfan Mafakheri, Stefano Frabboni, Ebrahim Karimi, and Robert W. Boyd, Holographic Generation of Highly Twisted Electron Beams, Phys. Rev. Lett. 114, 034801 (2015).

[28] Roeland Juchtmans, Armand Béché, Artem Abakumov, Maria Batuk, and Jo Verbeeck, Using electron vortex beams to determine chirality of crystals in transmission electron microscopy, Phys. Rev. B 91, 094112 (2015).

[29] V. G. Sala, D. D. Solnyshkov, I. Carusotto, T. Jacqmin, A. Lemaitre, H. Tercas, A. Nalitov, M. Abbarchi, E. Galopin, I. Sagnes, J. Bloch, G. Malpuech, and A. Amo, Spin-Orbit Coupling for Photons and Polaritons in Microstructures, Phys. Rev. X 5, 011034 (2015). 
[30] A. Anhauser, R. Wunenburger, and E. Brasselet, Acoustic Rotational Manipulation Using Orbital Angular Momentum Transfer, Phys. Rev. Lett. 109, 034301 (2012).

[31] Philip L. Marston, Axial radiation force of a bessel beam on a sphere and direction reversal of the force, J. Acoust. Soc. Am. 120, 3518 (2006).

[32] Diego Baresch, Jean-Louis Thomas, and Régis Marchiano, Observation of a single-beam gradient force acoustical trap for elastic particles: Acoustical tweezers, arXiv: 1411.1912.

[33] Alon Grinenko, Paul D. Wilcox, Charles R. P. Courtney, and Bruce W. Drinkwater, Proof of principle study of ultrasonic particle manipulation by a circular array device, Proc. R. Soc. A 468, 3571 (2012).

[34] Charles R. P. Courtney, Bruce W. Drinkwater, Christine E. M. Demore, Sandy Cochran, Alon Grinenko, and Paul D. Wilcox, Dexterous manipulation of microparticles using bessel-function acoustic pressure fields, Appl. Phys. Lett. 102, 123508 (2013).

[35] Antoine Riaud, Michael Baudoin, Jean-Louis Thomas, and Olivier Bou Matar, Cyclones and attractive streaming generated by acoustical vortices, Phys. Rev. E 90, 013008 (2014).

[36] G. Cincotti, A. Ciattoni, and C. Palma, Hermite-Gauss beams in uniaxially anisotropic crystals, IEEE J. Quantum Electron. 37, 1517 (2001).

[37] Dmitriy H. Zusin, Raman Maksimenka, Valerii V. Filippov, Ruslan V. Chulkov, Michel Perdrix, Olivier Gobert, and Alexander S. Grabtchikov, Bessel beam transformation by anisotropic crystals, J. Opt. Soc. Am. A 27, 1828 (2010).

[38] Tatyana Fadeyeva, Alexander Rubass, Yuriy Egorov, Alexander Volyar, and Jr. Grover Swartzlander, Quadrefringence of optical vortices in a uniaxial crystal, J. Opt. Soc. Am. A 25, 1634 (2008).

[39] Xiancong Lu and Lixiang Chen, Anisotropic dynamics of optical vortex-beam propagating in biaxial crystals: A numerical method based on asymptotic expansion, Opt. Express 21, 8493 (2013).

[40] Antoine Riaud, Jean-Louis Thomas, Michaël Baudoin, and Olivier Bou Matar, Taming the degeneration of Bessel beams at anisotropic-isotropic interface: Toward 3D control of confined vortical waves, arXiv:1508.07980.

[41] S. R. Fang, S. Y. Zhang, and Z. F. Lu, SAW focusing by circular-arc interdigital transducers on YZ-LiNbO3, IEEE Trans. Ultrason. Ferroelectr. Freq. Control 36, 178 (1989).

[42] Vincent Laude, Davy Gerard, Naima Khelfaoui, Carlos F. Jerez-Hanckes, Sarah Benchabane, and Abdelkrim Khelif, Subwavelength focusing of surface acoustic waves generated by an annular interdigital transducer, Appl. Phys. Lett. 92, 094104 (2008).

[43] Tsung-Tsong Wu, He-Tai Tang, Yung-Yu Chen, and P.-L. Liu, Analysis and design of focused interdigital transducers,
IEEE Trans. Ultrason. Ferroelectr. Freq. Control 52, 1384 (2005).

[44] Subramanian K. R. S. Sankaranarayanan and Venkat R. Bhethanabotla, Design of efficient focused surface acoustic wave devices for potential microfluidic applications, J. Appl. Phys. 103, 064518 (2008).

[45] R. C. Peach, A general Green function analysis for SAW devices, Proc. IEEE Ultrasonics Symposium 1, 221 (1995).

[46] V. P. Plessky and T. Thorvaldsson, Periodic Green's functions analysis of SAW and leaky SAW propagation in a periodic system of electrodes on a piezoelectric crystal, IEEE Trans. Ultrason. Ferroelectr. Freq. Control 42, 280 (1995).

[47] Donghai Qiao, Wen Liu, and P. M. Smith, General Green's functions for SAW device analysis, IEEE Trans. Ultrason. Ferroelectr. Freq. Control 46, 1242 (1999).

[48] V. Laude, C. F. Jerez-Hanckes, and S. Ballandras, Surface Green's function of a piezoelectric half-space, IEEE Trans. Ultrason. Ferroelectr. Freq. Control 53, 420 (2006).

[49] Mickaël Tanter, Jean-Louis Thomas, and Mathias Fink, Time reversal and the inverse filter, J. Acoust. Soc. Am. 108, 223 (2000).

[50] N. B. Piller and O. J. F. Martin, Extension of the generalized multipole technique to anisotropic medias, Opt. Commun. 150, 9 (1998).

[51] Juha Fagerholm, Ari T. Friberg, Juhani Huttunen, David P. Morgan, and Martti M. Salomaa, Angular-spectrum representation of nondiffracting $\mathrm{X}$ waves, Phys. Rev. E 54, 4347 (1996).

[52] Daniel Royer and Eugène Dieulesaint, Elastic Waves in Solids 1, Free and Guided Propagation (Springer, New York, 1996), Chap. 4.2.5.

[53] J. J. Campbell and W. R. Jones, Propagation of surface waves at the boundary between a piezoelectric crystal and a fluid medium, IEEE Trans. Sonics Ultrason. 17, 71 (1970).

[54] Olivier Bou Matar, Noura Gasmi, Huan Zhou, Marc Goueygou, and Abdelkrim Talbi, Legendre and Laguerre polynomial approach for modeling of wave propagation in layered magneto-electro-elastic media, J. Acoust. Soc. Am. 133, 1415 (2013).

[55] N. R. Heckenberg, R. McDuff, C. P. Smith, and A. G. White, Generation of optical phase singularities by computer-generated holograms, Opt. Lett. 17, 221 (1992).

[56] J.-L. Thomas and R. Marchiano, Pseudoangular Momentum and Topological Charge Conservation for Nonlinear Acoustical Vortices, Phys. Rev. Lett. 91, 244302 (2003).

[57] K. Volke-Sepulveda, A. O. Santillan, and R. R. Boullosa, Transfer of Angular Momentum to Matter from Acoustical Vortices in Free Space, Phys. Rev. Lett. 100, 024302 (2008).

[58] F. W. J. Olver, A further method for the evaluation of zeros of bessel functions and some new asymptotic expansions for zeros of functions of large order, Math. Proc. Cambridge Philos. Soc. 47, 699 (1951). 